\title{
PDF Analysis of Crystalline and Amorphous Materials on a Home Laboratory Diffractometer
}

\section{Joseph Ferrara ${ }^{1}$, Pierre Le Maguerès ${ }^{1}$, Christian Schürmann², Mathias Meyer ${ }^{3}$, Jakub Wojciechowski²,} Simon Bates ${ }^{1}$

\author{
${ }^{I}$ Rigaku Americas Corp, The Woodlands, United States of America; \\ ${ }^{2}$ Rigaku Europe SE, Frankfurt, Germany; \\ ${ }^{3}$ Rigaku Polska Sp. z o. o, Wroctaw, Poland; \\ joseph.ferrara@rigaku.com
}

There is no question that atomic pair distribution function analysis has had a profound impact on the analysis of crystalline and amorphous materials[1]. As a complement to the use of synchrotron sources for collecting PDF data, we have explored the use of home laboratory-based single crystal diffractometers to analyze both crystalline and amorphous materials. In order to generate the most useful reduced radial distribution functions, $\mathrm{G}(\mathrm{r})$, we have found it necessary to modify existing code in CrysAlis ${ }^{\text {Pro[ }[2]}$ and develop new code to generate G(r) data for refinement in PDFgui[3]. In this presentation we will explore the collection and analysis of total scattering data on both crystalline and amorphous materials with wavelengths readily available to home laboratory systems.

[1] Underneath the Bragg Peaks: Structural Analysis of Complex Materials, T. Egami and S. J. L. Billinge, Elsevier, Amsterdam, 2012, ISBN: 978-008-097133-9.

[2] Rigaku Oxford Diffraction, (2021), CrysAlisPro Software system, version 1.171.41.64, Rigaku Corporation, Wrocław, Poland.

[3] C. L. Farrow, P. Juhás, J. W. Liu, D. Bryndin, E. S. Božin, J. Bloch, Th. Proffen and S. J. L. Billinge, PDFfit2 and PDFgui: computer programs for studying nanostructure in crystals, J. Phys.: Condens. Matter, 19, 335219 (2007)

Keywords: PDF, total scattering 